\title{
Clinical Efficacy of Immunotherapy with Pd-1/Pd-L1 Inhibitors for the Treatment of Advanced Non-Small Cell Lung Cancer
}

\author{
Shengjie Yang ${ }^{1,2}$, Xiao Ma ${ }^{4}$, Wenjie Zhang ${ }^{2,3}$, Qing Chen ${ }^{1,2}$, Qisen Guo ${ }^{2, *}$ \\ ${ }^{1}$ School of Medicine and Life Sciences, University of Jinan-Shandong, Academy of Medical Sciences, Jinan, \\ China \\ ${ }^{2}$ Shandong Cancer Hospital and Institute, Shandong First Medical University and Shandong Academy of \\ Medical Sciences, Jinan, China \\ ${ }^{3}$ Department of Oncology, Weifang Medical University, Weifang, Shandong, China \\ ${ }^{4}$ Department of Internal Medicine, Zhangqiu People’s Hospital, Jinan, China \\ Email: guoqs369369@163.com \\ *Corresponding Author
}

Keywords: Lung cancer, Pd-1, Pd-11, Pd-1/pd-l1 inhibitors, Effectiveness, Survival

\begin{abstract}
Immunotherapeutics like programmed cell death protein 1 (PD-1)/programmed death-ligand 1 (PD-L1) inhibitors effectively control non-small cell lung cancer (NSCLC) in clinical trials. This study assessed the association of different clinicopathological parameters with the treatment effectiveness and prognosis of NSCLC patients in a real-world setting. Seventy-five patients received PD-1/PD-L1 inhibitors and were evaluated for overall survival (OS), progression-free survival (PFS), objective response rate (ORR), and disease control rate (DCR). Eighteen patients reached a partial response (PR) (24\%), 45 had stable disease (SD) (60\%), and 12 had progressive disease (PD) (16\%), resulting in an ORR of $24 \%$ and a DCR of $84 \%$. For the 11 PD-L1-positive patients, the ORR was 36.36\%. Moreover, 16 patients (21\%) had died and 34 (45\%) had SD at the last follow-up, leading to a PFS of 8.3 months (95\% confidence interval (CI): 6.812.2 months) and an OS of 17 months (95\%CI: 16.7-26.5 months). The Eastern Cooperative Oncology Group (ECOG) score was associated with the treatment-related PFS and OS. The factors associated with the prognosis were ECOG score, line of immunotherapy, tobacco smoking, PD-L1 positivity, and tumor pathology. This study confirmed the clinical effectiveness of PD-1/PD-L1 inhibitor immunotherapy against advanced NSCLC.
\end{abstract}

\section{Introduction}

Lung cancer remains the most significant worldwide health problem and causes high morbidity and mortality rates in both men and women (1). Although the implementation of tobacco cessation programs has significantly reduced the incidence of lung cancer in the USA and other countries, the incidence of lung cancer is on the rise throughout the world (2-4). Lung cancer can be histologically divided into small cell lung cancer and non-small cell lung cancer (NSCLC), the latter of which comprises up to $80-85 \%$ of all lung cancer cases (5). In recent years, novel therapies to treat and control advanced NSCLC have been implemented and have improved the survival of NSCLC patients (6-9). Specifically, in addition to the traditional platinum-based dual-drug chemotherapy, many NSCLC patients with gene mutations (such as epidermal growth factor receptor (EGFR), anaplastic lymphoma kinase (ALK), mesenchymal-epithelial transition factor, and ROS proto-oncogene 1, receptor tyrosine kinase) can further prolong their progression-free survival (PFS) and overall survival (OS) with molecular targeted drugs (10). However, although the molecular targeted drugs are effective, not all NSCLCs have gene mutations; in addition, even with gene mutations, some molecular targeted drugs that used to be effective no longer work due to acquired resistance (11). Thus, in the face of such a large number of NSCLC cases, there is an urgent need to further discover and identify novel approaches and strategies to more effectively control and treat 
NSCLC. Interestingly, the emergence of immune checkpoint inhibitors provides promise; indeed, programmed cell death protein 1 (PD-1) and programmed death-ligand 1 (PD-L1) are the most widely used immune checkpoint targets, together with other immune checkpoints, like cytotoxic T-lymphocyte-associated protein 4, T cell immunoglobulin and mucin domain-containing protein 3, lymphocyte-activation gene 3 , and $\mathrm{T}$ cell immunoreceptor with $\mathrm{Ig}$ and immunoreceptor tyrosine-based inhibitory motif domains. Furthermore, National Comprehensive Cancer Network data have shown that nivolumab can be used as the second-line therapy for patients with nonsensitive mutant NSCLC, while pembrolizumab can be used as the second-line therapy for patients with more than 1\% PD-L1-positive NSCLC or as the first-line treatment for patients with $50 \%$ or more PD-L1-positive NSCLC (12-17). To date, the results of different clinical trials show that immune checkpoint inhibitors are effective (12-17). However, such randomized controlled trials have strict screening criteria to recruit patients; for example, older patients, patients with Eastern Cooperative Oncology Group (ECOG) scores of 2 or more, or patients with brain metastasis are less likely to be enrolled into these clinical trials. Thus, in this study, we assessed real-world data to overcome the deficiencies of some randomized controlled trials by comprehensively analyzing the data on immune checkpoint inhibitors for the treatment of advanced NSCLC patients at the Shandong Cancer Hospital as well as the different clinicopathological parameters for association with the prognosis. This study will provide clinicians with a reference to help them better utilize anti-NSCLC therapy in the clinic.

\section{Materials and Methods}

\subsection{Patients and Data Collection}

This study enrolled 77 NSCLC patients who received PD-1/PD-L1 inhibitors between August 2017 and November 2019 in Shandong Cancer Hospital (Jinan, China). All patients were diagnosed histologically with NSCLC and classified according to the tumor, lymph node, and metastasis (TNM) staging system for lung cancer (18). This study was approved by the Ethics Committee of the Shandong Cancer Hospital and followed the Helsinki Declaration as well as the Guidelines for Good Clinical Practice. Informed consent was obtained from all participants before enrollment into this study.

Clinicopathological data, including sex; age at diagnosis; TNM stage; pathological type of lung cancer; status of the EGFR mutations; tumor molecular pathologies, like PD-L1 expression; ECOG score; tobacco smoking history; tumor brain metastasis; treatment plan; time of tumor progression; number of treatment lines completed before starting immunotherapy; immunotherapy cycle; immune-related side effects; as well as laboratory tests, like routine blood tests, liver and renal function, tumor markers, myocardial zymogram, thyroid function, and imaging examinations (computed tomography, bone scan, skull magnetic resonance imaging, or positron emission tomography-computed tomography), were collected from the medical records of the patients.

\subsection{Treatment Plans}

A total of 75 patients received immunotherapy, among whom 34 received immunotherapy as the first-line therapy, 17 received immunotherapy as the second-line therapy, and 24 received immunotherapy as the third-line or above therapy. The routine blood as well as liver, kidney, thyroid, and heart function tests were carried out before each treatment cycle, while computed tomography imaging was taken every three cycles, and the treatment cycles continued until intolerable toxicity or disease progression.

\subsection{Treatment Evaluations}

During the follow-up, two patients who received immunotherapy as the third-line or above therapy were lost; thus, our data analysis excluded them, resulting in 75 patients in this study. The effectiveness of the treatment was evaluated according to the evaluation criteria of solid tumor efficacy (RECIST1.1) for complete response (CR), partial response (PR), stable disease (SD), or 
progressive disease (PD). The objective response rate (ORR) was defined as the total of $\mathrm{CR}+\mathrm{PR}$ patients of the total 75 patients, while the disease control rate (DCR) was defined as the total of $\mathrm{CR}+\mathrm{PR}+\mathrm{SD}$ patients of these 75 patients. The PFS referred to the duration from the first PD-1/PD-L1 inhibitor treatment to PD or death, while the OS refers to the duration from the first PD-1/PD-L1 inhibitor treatment to death or the last follow-up.

\subsection{Statistical Analysis}

The categorical variables were summarized as percentages, while the continuous variables were summarized as median values. The data were statistically analyzed using SPSS22.0 software (SPSS, Chicago, IL, USA). Fisher's exact test was performed to analyze the short-term efficacy and clinical features. The PFS and OS were analyzed using the Kaplan-Meier method and the log-rank test. P < 0.05 was considered to be statistically significant. Due to the small sample size and the low number of classification variables, this study also utilized the random forest method, random resampling technique (bootstrap aggregation), and random subspace method to construct multiple decision trees for the final classification results. The random forest was used to rank the importance of variables in regression or classification problems naturally; this step greatly reduced the limitations of the traditional Fisher's exact test methods for the categorical data.

\section{Results}

\subsection{Baseline Characteristics}

Table 1 Baseline Characteristics of the Nsclc Patients $(n=75)$

\begin{tabular}{|c|c|}
\hline Variable & N (\%) \\
\hline Age (median, range) & $61(27-87)$ \\
\hline \multicolumn{2}{|l|}{ Sex } \\
\hline Male & $51(68)$ \\
\hline Female & $24(32)$ \\
\hline \multicolumn{2}{|l|}{ Pathological type } \\
\hline 0 & $22(29.3)$ \\
\hline 1 & $53(70.7)$ \\
\hline \multicolumn{2}{|l|}{ ECOG PS score } \\
\hline 0 & $20(26.7)$ \\
\hline 1 & $30(40)$ \\
\hline 2 & $16(21.3)$ \\
\hline 3 & $7(9.3)$ \\
\hline 4 & $2(2.7)$ \\
\hline \multicolumn{2}{|l|}{ Stage } \\
\hline III & $10(13.3)$ \\
\hline IV & 65 (86.7) \\
\hline \multicolumn{2}{|l|}{ Smoking history } \\
\hline Never & 40 (53.3) \\
\hline Yes & 35 (46.7) \\
\hline \multicolumn{2}{|c|}{ Line of immunotherapy } \\
\hline 1 & 34 (45.3) \\
\hline 2 & $17(22.7)$ \\
\hline$\geqslant 3$ & $24(32)$ \\
\hline \multicolumn{2}{|l|}{ PD-L1 status } \\
\hline Positive & $11(14.7)$ \\
\hline Negative/unknown & $64(85.3)$ \\
\hline \multicolumn{2}{|l|}{ Mutation of EGFR } \\
\hline Positive & $10(13.3)$ \\
\hline Negative/unknown & $65(86.7)$ \\
\hline \multicolumn{2}{|l|}{ Brain metastasis } \\
\hline Positive & $21(28)$ \\
\hline
\end{tabular}

In this study, we enrolled a total of 77 NSCLC patients who were treated with PD-1/PD-L1 inhibitors. However, during the follow-up, 2 patients were lost and excluded from our data analysis, 
resulting in 75 patients. Among these 75 patients, the median age was 61 years old (range: 27-87 years old); 9 patients (12\%) were older than 70 years old, and 3 patients (4\%) were older than 75 years old. The patients included 51 males (68\%) and 24 females (32\%). There were 53 cases of adenocarcinoma $(70.7 \%)$ and 22 cases of squamous cell carcinoma (29.3\%). Nearly half of the patients had a history of tobacco smoking (46.7\%), and most had pathological stage IV disease (86.7\%). Thirty-four patients (45.3\%) received the immunotherapy as the first-line treatment; 21 patients (12.8\%) had craniocerebral metastasis before immunotherapy; 10 patients had EGFR-sensitive mutations; and the rest had no gene mutations or were not tested. Moreover, 11 patients were PD-L1-positive before immunotherapy, while the others were not tested or the results were negative (Table 1). Of all 75 patients included in this study, 18 had a PR (24\%), 45 had SD (60\%), 12 had PD (16\%), and no patients achieved a CR (0\%). The ORR was 24\%, and the DCR was $84 \%$. Of the nine patients aged 70 years or older, two patients achieved a PR and two patients had SD; while among the three patients aged 75 years or older, one achieved a PR and one had SD. No grade III or IV adverse effects occurred in this cohort of patients. Of the 21 patients with craniocerebral metastasis, we found that the immunotherapy resulted in a PR for 4 patients, SD for 12 patients, and PD for 5 patients, according to the images of the intracranial lesions. The ORR for patients with intracranial metastasis who received immunotherapy was $19.05 \%$ and the DCR was 76.19\%. Moreover, of the 10 patients with EGFR-sensitive mutations, 4 patients showed a PR, 4 patients had SD, and 2 patients had PD. For the 11 PD-L1-positive patients, the ORR reached $36.36 \%$. The data on other clinicopathological parameters, including sex, age, pathological type, ECOG score, pathological stage, smoking history, treatment line, PD-L1 expression, EGFR gene test results, and brain metastasis, are shown in Table 2.

Table 2 Association of Clinicopathological Features with the Short-Term Effectiveness of Pd-1/Pd-L1 Inhibitor Therapy in 75 Nsclc Patients

\begin{tabular}{|c|c|c|c|c|c|c|c|c|}
\hline & $\mathrm{N}$ & PD & PR & SD & ORR (\%) & ORR (P-value) & DCR (\%) & DCR (P-value) \\
\hline \multicolumn{9}{|l|}{ Sex } \\
\hline Male & 51 & 7 & 13 & 31 & 25.49 & \multirow[t]{2}{*}{0.66} & 86.27 & \multirow[t]{2}{*}{0.50} \\
\hline Female & 24 & 5 & 5 & 14 & 20.83 & & 79.17 & \\
\hline \multicolumn{9}{|l|}{ Age (years old) } \\
\hline$<70$ & 66 & 10 & 16 & 40 & 60.61 & \multirow[t]{2}{*}{0.94} & 40.24 & \multirow[t]{2}{*}{0.95} \\
\hline$\geqslant 70$ & 9 & 2 & 2 & 5 & 55.56 & & 77.78 & \\
\hline \multicolumn{9}{|l|}{ Pathological type } \\
\hline 0 & 22 & 4 & 4 & 14 & 18.18 & \multirow[t]{2}{*}{0.44} & 81.82 & \multirow[t]{2}{*}{0.73} \\
\hline 1 & 53 & 8 & 14 & 31 & 26.42 & & 84.91 & \\
\hline \multicolumn{9}{|l|}{ ECOG PS score } \\
\hline 0 & 20 & 1 & 6 & 13 & 30 & \multirow[t]{5}{*}{0.20} & 95 & \multirow[t]{5}{*}{0.096} \\
\hline 1 & 30 & 5 & 9 & 16 & 30 & & 83.33 & \\
\hline 2 & 16 & 4 & 1 & 11 & 6.25 & & 75 & \\
\hline 3 & 7 & 1 & 2 & 4 & 28.57 & & 85.71 & \\
\hline 4 & 2 & 1 & 0 & 1 & 0 & & 50 & \\
\hline \multicolumn{9}{|l|}{ Stage } \\
\hline III & 10 & 2 & 3 & 5 & 30 & \multirow[t]{2}{*}{0.69} & 80 & \multirow[t]{2}{*}{0.65} \\
\hline IV & 65 & 10 & 15 & 40 & 23.08 & & 84.62 & \\
\hline \multicolumn{9}{|l|}{ Smoking history } \\
\hline Never & 40 & 7 & 7 & 26 & 17.5 & \multirow[t]{2}{*}{0.15} & 82.5 & \multirow[t]{2}{*}{0.70} \\
\hline Yes & 35 & 5 & 11 & 19 & 31.43 & & 85.71 & \\
\hline \multicolumn{9}{|c|}{ Line of immunotherapy } \\
\hline 1 & 34 & 4 & 7 & 23 & 20.59 & \multirow[t]{3}{*}{0.72} & 88.24 & \multirow[t]{3}{*}{0.42} \\
\hline 2 & 17 & 4 & 4 & 9 & 23.53 & & 76.47 & \\
\hline$\geqslant 3$ & 24 & 4 & 7 & 13 & 29.17 & & 83.33 & \\
\hline \multicolumn{9}{|l|}{ PD-L1 status } \\
\hline Positive & 11 & 0 & 4 & 7 & 36.36 & \multirow[t]{2}{*}{0.44} & 100 & \multirow[t]{2}{*}{0.19} \\
\hline Negative/unknown & 64 & 12 & 14 & 38 & 21.88 & & 81.25 & \\
\hline \multicolumn{9}{|l|}{ Mutation of EGFR } \\
\hline Positive & 10 & 2 & 4 & 4 & 40 & \multirow[t]{2}{*}{0.23} & 80 & \multirow[t]{2}{*}{0.65} \\
\hline Negative/unknown & 65 & 10 & 14 & 41 & 21.54 & & 84.62 & \\
\hline Brain metastasis & & & & & & & & \\
\hline Positive & 21 & 5 & 4 & 12 & 19.05 & 0.53 & 76.19 & 0.29 \\
\hline Negative & 54 & 7 & 14 & 33 & 25.93 & & 87.04 & \\
\hline
\end{tabular}


As of the last follow-up (November 30, 2019), 16 patients (21.33\%) had died and 34 (45.33\%) had no disease progression (the longest PFS was 19.1 months and the longest OS was 99.3 months), while the median OS and PFS of the 75 patients were 8.3 months (95\% confidence interval (CI): 6.8-12.2 months) and 17 months (95\%CI: 16.65-26.47 months), respectively (Figure 1A-B).

A

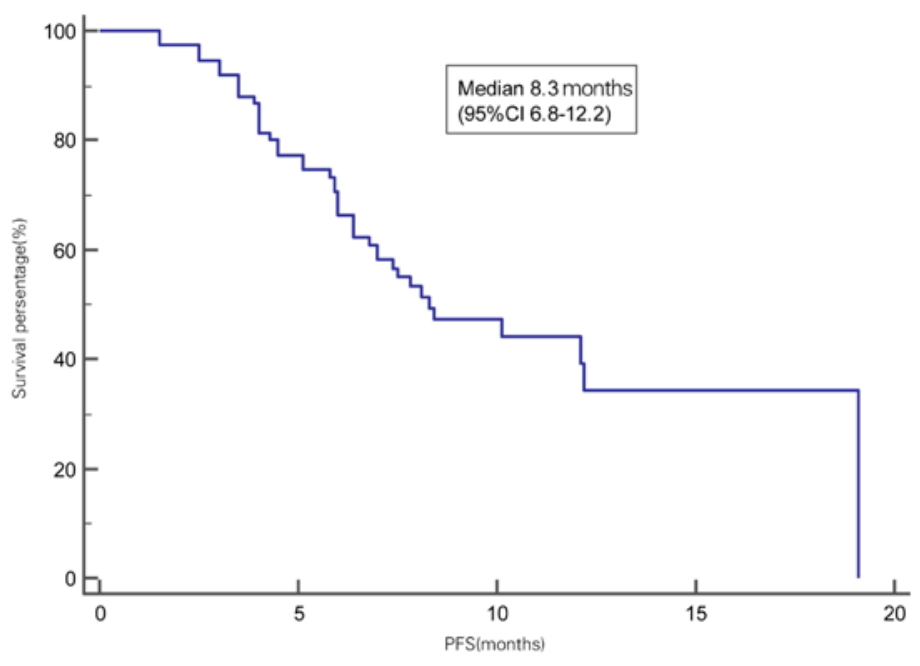

B

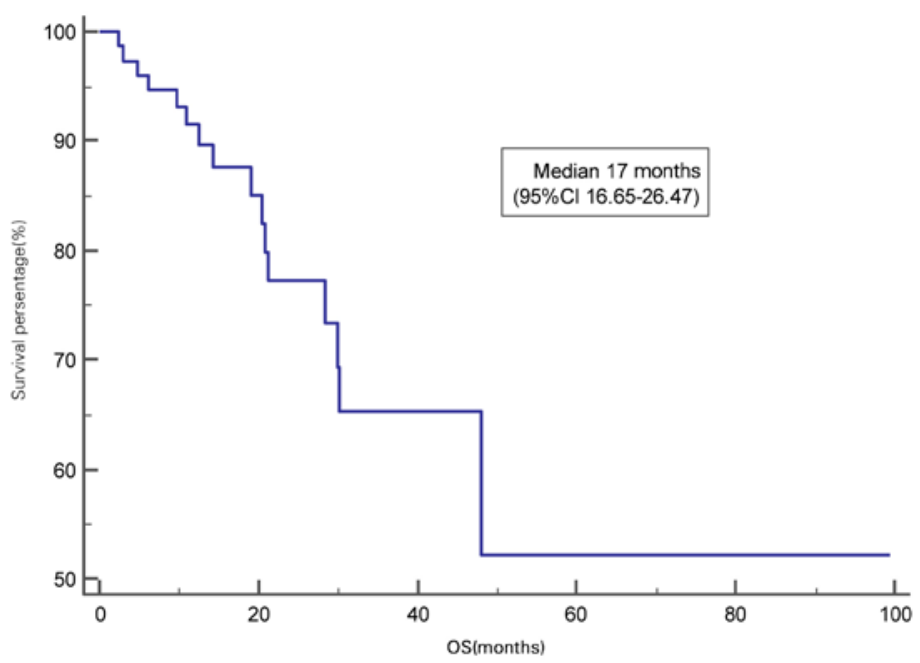

Fig.1 Kaplan-Meier Survival Curves of the Progression-Free Survival (Pfs) and Overall Survival (Os) of 75 Patients. a, Pfs. B, Os.

Furthermore, the clinicopathological factors were associated with the PFS. We found that the ECOG score was significantly correlated with the PFS $(\mathrm{p}=0.003$; Figure $2 \mathrm{~A})$, but not with the patient age $(p=0.84)$, sex $(p=0.311)$, pathological stage $(p=0.59)$, pathological type $(p=0.24)$, immunotherapy line $(p=0.93)$, brain metastasis $(p=0.20)$, EGFR mutation status $(p=0.068)$, or PD-L1 status $(\mathrm{p}=0.10)$. After that, we performed multivariate analyses, and our data showed that only the ECOG score predicted the PFS in a statistically significant manner (Table 3). Similarly, we also associated the clinicopathological factors with OS and found that the ECOG score was significantly associated with the OS (Figure 2B) but not the other clinicopathological factors, i.e., the patient age $(p=0.97)$, sex $(p=0.41)$, stage $(p=0.72)$, pathological type $(p=0.26)$, immunotherapy line $(p=0.23)$, brain metastasis $(p=0.61)$, EGFR mutation status $(p=0.50)$, or PD-L1 status ( $p=0.93)$. Cox multivariate regression analysis showed that only the ECOG score could independently predict the OS in a statistically significant manner (Table 4). 
Table 3 Cox Multivariate Regression Analysis of Pfs

\begin{tabular}{|l|l|l|l|l|}
\hline \multicolumn{3}{|l|}{} \\
\hline Variable & P-value & HR & $95 \%$ CI \\
\cline { 4 - 6 } & & & & \\
\hline Stage & 0.86 & 0.906 & 0.29 & 2.834 \\
\hline Age & 0.92 & 0.951 & 0.348 & 2.603 \\
\hline ECOG score (1 vs. 0) & 0.005 & 0.085 & 0.015 & 0.48 \\
\hline ECOG score (2 vs. 0) & 0.094 & 0.253 & 0.051 & 1.262 \\
\hline ECOG score (3 vs. 0) & 0.29 & 0.415 & 0.079 & 2.183 \\
\hline ECOG score (4 vs. 0) & 0.46 & 0.494 & 0.074 & 3.3 \\
\hline PD-L1 status & 0.059 & 3.333 & 0.958 & 11.605 \\
\hline Line of immunotherapy (2 vs. 1) & 0.21 & 1.731 & 0.721 & 4.157 \\
\hline Line of immunotherapy ( $\geqslant 3$ vs. 1) & 0.25 & 1.737 & 0.666 & 4.531 \\
\hline
\end{tabular}

Table 4 Cox Multivariate Regression Analysis of Overall Survival

\begin{tabular}{|l|l|l|l|l|}
\hline \multicolumn{2}{|l|}{ Variable } & P-value & HR & $95 \%$ CI \\
\cline { 3 - 5 } & & & \\
\hline Stage & 0.49 & 2.287 & 0.212 & 24.663 \\
\hline Age & 0.22 & 3.687 & 0.455 & 29.873 \\
\hline ECOG score (1 vs. 0) & 0.002 & 0.01 & 0.001 & 0.174 \\
\hline ECOG score (2 vs. 0) & 0.003 & 0.035 & 0.004 & 0.32 \\
\hline ECOG score (3 vs. 0) & 0.032 & 0.087 & 0.009 & 0.813 \\
\hline ECOG score (4 vs. 0) & 0.19 & 0.211 & 0.02 & 2.19 \\
\hline PD-L1 status & 0.65 & 1.669 & 0.183 & 15.237 \\
\hline Line of immunotherapy (2 vs. 1) & 0.20 & 3.006 & 0.558 & 16.198 \\
\hline Line of immunotherapy ( $\geqslant 3$ vs. 1) & 0.77 & 0.779 & 0.145 & 4.174 \\
\hline
\end{tabular}

A

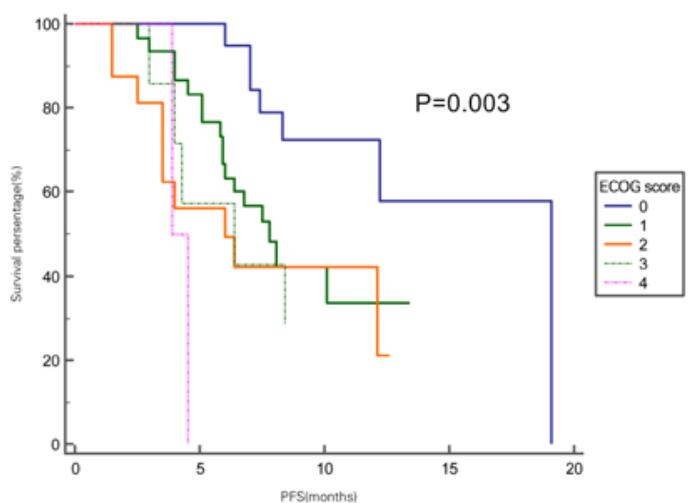

B

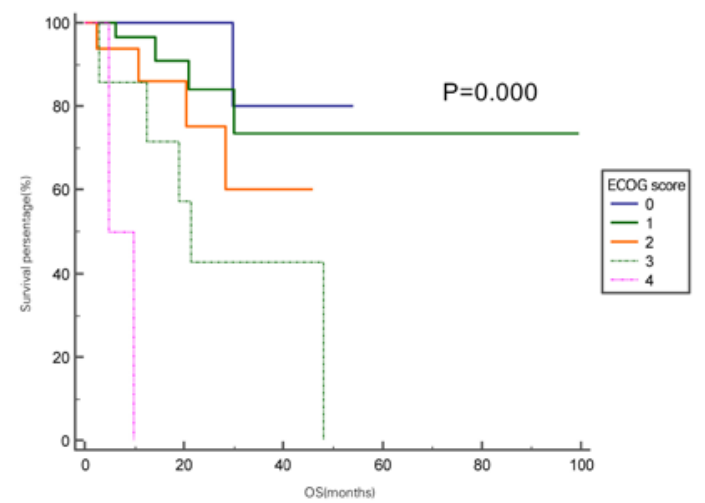

Fig.2 Kaplan-Meier Survival Curves of Progression-Free Survival (Pfs) and Overall Survival (Os) after Comparison with the Eastern Cooperative Oncology Group (Ecog) Scores of the 75 Patients Included in This Study. a, Pfs. B, Os. 


\subsection{Random Forest Analysis of Effects of the Pd-1/Pd-L1 Inhibitors on Nsclc}

Next, due to the small sample size of this study, we performed random forest analysis of the data to predict the factors that may affect the OS of NSCLC patients after immunotherapy. Specifically, the OS duration was used as the dependent variable; while the patient sex, pathological type, age, and other variables were taken as independent variables to construct the prediction model. We found that the error rate of the out-of-bag estimation model was $13.33 \%$ and the prediction accuracy was $86.67 \%$. The accuracy of the retrograde prediction of the original samples reached $100 \%$, indicating that there was a stable correlation between the predictive variables of this study and the survival state of patients after immunotherapy (Table 5). The higher the score was, the greater the importance to the survival of patients.

Table 5 the Importance of the Factors Associated with the Effectiveness of Pd-1/Pd-L1 Inhibitor Therapy in 75 Nsclc Patients

\begin{tabular}{|l|l|l|}
\hline Order of importance & Variable & Importance score \\
\hline 1 & ECOG score & 3.49 \\
\hline 2 & Line of immunotherapy & 1.76 \\
\hline 3 & Smoking history & 1.46 \\
\hline 4 & PD-L1 status & 0.52 \\
\hline 5 & Pathological type & 0.48 \\
\hline 6 & Sex & 0.33 \\
\hline 7 & Age & 0.32 \\
\hline 8 & Brain metastasis & 0.31 \\
\hline 9 & EGFR mutation & 0.30 \\
\hline 10 & Stage & 0.21 \\
\hline
\end{tabular}

\section{Discussion}

In the current study, we evaluated the effectiveness of PD-1/PD-L1 inhibitor therapy in 75 NSCLC patients. Our analysis of the short-term curative effects of the PD-1/PD-L1 inhibitors on NSCLC showed 18 patients with a PR (24\%), 45 with SD (60\%), and 12 with PD (16\%). The ORR was $24 \%$ and the DCR was $84 \%$, while the effectiveness of the PD-1/PD-L1 inhibitors on the 11 PD-L1-positive patients reached 36.36\% of the ORR. Our analysis of the long-term curative effects of the PD-1/PD-L1 inhibitors on NSCLC showed that the median PFS was 8.3 months (95\%CI: 6.8-12.2 months) and the median OS was 17 months (95\%CI: 16.65-26.67). The immunotherapy was more effective in patients with good physiques and lower ECOG scores, whereas it was equally effective in older patients ( $\geqslant 70$ years) and younger patients. The treatment was the most effective for patients with PD-L1-positive NSCLC as well as patients with EGFR mutations. The immunotherapy might also help control NSCLC brain metastasis. Thus, this study revealed the clinical effectiveness of PD-1/PD-L1 inhibitor immunotherapy against advanced NSCLC.

Indeed, lung cancer is the leading cause of cancer-related deaths in the world (1). The current immunotherapy with PD-1/PD-L1 inhibitors provides a novel approach to effectively control NSCLC in the clinic. As is well known, the physical fitness of lung cancer patients is a very important prognostic factor (19); for example, NSCLC patients with an ECOG score of $\leqslant 2$ benefit the most from antineoplastic therapy. However, many patients with a score of 3-4 still require antineoplastic therapy. In the current study, we evaluated such patients after PD-1/PD-L1 inhibitor immunotherapy and found that the ECOG score was an independent factor to predict the PFS and OS, i.e., the lower the ECOG score was, the higher the PFS and OS were after the immunotherapy, which is in line with the clinical findings; thus, our data indicate that patients with good physiques could benefit from the treatment.

The incidence of lung cancer increases with age (20), with the diagnosis of lung cancer peaking in those aged 65 years or older. Therefore, with the population aging, the lung cancer incidence is expected to rise. It is important to explore the survival benefit of immunotherapy in elderly patients because an increase in age is usually accompanied with a decline in functions of the immune system, 
especially the $\mathrm{T}$ cell-mediated immune defense system, resulting in a poor efficacy of immune checkpoint inhibitors for the treatment of elderly patients (21). Other studies have shown that aging may not affect the effectiveness of immunotherapy (22-24). In the current study, we included nine NSCLC patients aged over 70 years old and found that two of them reached a PR and five reached $\mathrm{SD}$, indicating that there was no significant difference in the benefit of such a treatment in older patients compared with younger patients. To the best of our knowledge, PD-1/PD-L1 inhibitors are able to block the activity of the PD-1 and PDL1 immune checkpoint proteins that are expressed on the surface of different tumor cells; thus, they are used as a front-line treatment for several cancer types (25). Specifically, PD-L1 on tumor cells interacts with PD-1 protein on T-lymphocytes and reduces the functions and signaling of the T-cell-mediated immune responses; therefore, it prevents T-lymphocytes from attacking the tumor cells (26). In this regard, a PD-1/PD-L1 inhibitor should block such an interaction of PD-L1 with the PD-1 receptor and enhance antitumor immune reactions; such a drug has been used successfully in the clinic to treat various human cancers, including NSCLC (27). Although elderly patients may suffer from low immune responses and the effectiveness of PD-1/PD-L1 inhibitors is thought to be low, our current data showed that the patient age ranked as number 7 (0.32) for the effectiveness of the PD-1/PD-L1 inhibitor after sex. However, our sample size was small and further study is needed to verify our current data. Moreover, two different clinical trials $(16,17)$ have revealed the importance of PD-L1 expression for the effectiveness of PD-1/PD-L1 inhibitor immunotherapy against advanced NSCLC. Nevertheless, it is undeniable that it did not matter whether PD-L1 was expressed or not as all patients could benefit from the immunotherapy; thus, we speculate that in clinical practice, as long as the patients can tolerate the treatment, we should treat them with immune checkpoint inhibitors for antitumor therapy.

NSCLCs frequently metastasize to the brain, accounting for approximately 40-50\% of NSCLC patients (28), and the prognosis of such patients is also poor (29). In the current study of 75 patients, there were 21 patients with brain metastasis; after the treatment, 5 reached PD, 4 reached a PR, and 12 had SD. Although the sample size was small, our data indicate that these NSCLC patients with brain metastasis also benefited from the immunotherapy. These findings are consistent with a previously published report (30). Moreover, the results of previous clinical trials $(14,31,32)$ have shown that NSCLC patients with EGFR/ALK mutations are not sensitive to immunotherapy. The common reason is that patients with EGFR/ALK-sensitive mutations cannot easily produce novel tumor antigens, resulting in a low tumor mutation load. Our current study of 10 patients with EGFR-sensitive mutations showed that 4 patients reached a PR, 4 reached SD, and 2 had PD. Comparing patients with or without EGFR-sensitive mutations, the ORR in patients with EGFR-sensitive mutations was higher than that of patients without EGFR-sensitive mutations, although it did not reach statistical significance. Further study will confirm this finding.

In addition, our current data showed that the ECOG score was the most statistically significant index that was associated with the survival of patients after the treatment. There was no significant association with the patient age or sex, tumor stage, pathological type, line of immunotherapy, brain metastasis, EGFR mutation status, or PD-L1 status. However, our current study does have some limitations. For example, the sample size was small and did not meet the requirements for survival analysis. To overcome the limitations of the traditional statistical classification method, e.g., when the model is collinear with independent variables, the estimation of model parameters is unstable. We further included the impact of random forest predictive variables on the OS. Our data revealed that the important factors affecting the survival rate were as follows (from the most to least): ECOG score, line of immunotherapy, smoking history, PD-L1 status, pathological type, sex, age, brain metastasis, EGFR mutations, and tumor stage. This list provides an intuitive and simple variable model for selecting people who may be more suitable for immunotherapy.

\section{References}

[1] Bray F, Ferlay J, Soerjomataram I, et al. Global cancer statistics 2018: GLOBOCAN estimates of incidence and mortality worldwide for 36 cancers in 185 countries. CA Cancer J Clin, Vol. 68, 
No.6, pp. 394-424, 2018.

[2] Chen W, Zheng R, Baade PD, et al. Cancer statistics in China, 2015. CA Cancer J Clin, Vol. 66, No.2, pp. 115-132, 2016.

[3] Siegel RL, Miller KD, Jemal A. Cancer statistics, Vol. 70, No. 1, pp. 7-30, 2020.

[4] Bade BC, Dela Cruz CS. Lung Cancer 2020: Epidemiology, Etiology, and Prevention. Clin Chest Med, Vol. 41, No. 1, pp. 1-24, 2020.

[5] Jamal-Hanjani M, Wilson GA, McGranahan N, et al. Tracking the Evolution of Non-Small-Cell Lung Cancer. N Engl J Med, Vol. 376, No. 22, pp. 2109-2121, 2017.

[6] Antonia SJ, Villegas A, Daniel D, et al. Durvalumab after Chemoradiotherapy in Stage III Non-Small-Cell Lung Cancer. N Engl J Med, Vol. 377, No.20, pp. 1919-1929, 2017.

[7] Forde PM, Chaft JE, Smith KN, et al. Neoadjuvant PD-1 Blockade in Resectable Lung Cancer. N Engl J Med, Vol. 378, No. 21, pp. 1976-1986, 2018.

[8] Ramalingam SS, Vansteenkiste J, Planchard D, et al. Overall Survival with Osimertinib in Untreated, EGFR-Mutated Advanced NSCLC. N Engl J Med, Vol. 382, No.1, pp. 41-50, 2020.

[9] de Koning HJ, van der Aalst CM, de Jong PA, et al. Reduced Lung-Cancer Mortality with Volume CT Screening in a Randomized Trial. N Engl J Med, Vol. 382, No. 6, pp. 503-513, 2020.

[10] Yang JC, Wu YL, Schuler M, et al. Afatinib versus cisplatin-based chemotherapy for EGFR mutation-positive lung adenocarcinoma (LUX-Lung 3 and LUX-Lung 6): analysis of overall survival data from two randomised, phase 3 trials. Lancet Oncol, Vol. 16, No. 2, pp. 141-151, 2015.

[11] Zeng L, Xiao L, Jiang W, et al. Investigation of efficacy and acquired resistance for EGFR-TKI plus bevacizumab as first-line treatment in patients with EGFR sensitive mutant non-small cell lung cancer in a Real world population. Lung Cancer, No.141, pp. 82-88, 2020.

[12] Brahmer J, Reckamp KL, Baas P, et al. Nivolumab versus Docetaxel in Advanced Squamous-Cell Non-Small-Cell Lung Cancer. N Engl J Med, Vol. 373, No. 2, PP. 123-135, 2015.

[13] Gettinger S, Rizvi NA, Chow LQ, et al. Nivolumab Monotherapy for First-Line Treatment of Advanced Non-Small-Cell Lung Cancer. J Clin Oncol, Vol. 34, No. 25, pp. 2980-2987, 2016.

[14] Herbst RS, Baas P, Kim DW, et al. Pembrolizumab versus docetaxel for previously treated, PD-L1-positive, advanced non-small-cell lung cancer (KEYNOTE-010): a randomised controlled trial. Lancet, Vol. 387, No. 10027, pp. 1540-1550, 2016.

[15] Langer CJ, Gadgeel SM, Borghaei H, et al. Carboplatin and pemetrexed with or without pembrolizumab for advanced, non-squamous non-small-cell lung cancer: a randomised, phase 2 cohort of the open-label KEYNOTE-021 study. Lancet Oncol, Vol. 17, No.11, pp. 1497-1508, 2016.

[16] Mok TSK, Wu YL, Kudaba I, et al. Pembrolizumab versus chemotherapy for previously untreated, PD-L1-expressing, locally advanced or metastatic non-small-cell lung cancer (KEYNOTE-042): a randomised, open-label, controlled, phase 3 trial. Lancet, Vol. 393, No.10183, pp. 1819-1830, 2019.

[17] Reck M, Rodriguez-Abreu D, Robinson AG, et al. Pembrolizumab versus Chemotherapy for PD-L1-Positive Non-Small-Cell Lung Cancer. N Engl J Med, Vol. 375, No.19, pp. 1823-1833, 2016.

[18] Goldstraw P, Chansky K, Crowley J, et al. The IASLC Lung Cancer Staging Project: Proposals for Revision of the TNM Stage Groupings in the Forthcoming (Eighth) Edition of the TNM Classification for Lung Cancer. J Thorac Oncol, Vol. 11, No.1, pp. 39-51, 206.

[19] Huemer F, Lang D, Westphal T, et al. Baseline Absolute Lymphocyte Count and ECOG Performance Score Are Associated with Survival in Advanced Non-Small Cell Lung Cancer 
Undergoing PD-1/PD-L1 Blockade. J Clin Med, Vol. 8, No.7, 2019.

[20] Bray F, Jemal A, Grey N, et al. Global cancer transitions according to the Human Development Index (2008-2030): a population-based study. Lancet Oncol, Vol. 13, No.8, pp. 790-801, 2012.

[21] Tomihara K, Curiel TJ, Zhang B. Optimization of immunotherapy in elderly cancer patients. Crit Rev Oncog, Vol. 18, No.6, pp. 573-583, 2013.

[22] Elias R, Morales J, Presley C. Checkpoint Inhibitors for Non-Small Cell Lung Cancer Among Older Adults. Curr Oncol Rep, Vol. 19, No.9, pp.62, 2017.

[23] Ferrara R, Mezquita L, Auclin E, et al. Immunosenescence and immunecheckpoint inhibitors in non-small cell lung cancer patients: Does age really matter? Cancer Treat Rev, No.60, pp.60-68, 2017.

[24] Lichtenstein MRL, Nipp RD, Muzikansky A, et al. Impact of Age on Outcomes with Immunotherapy in Patients with Non-Small Cell Lung Cancer. J Thorac Oncol, Vol. 14, No.3, pp. 547-552, 2019.

[25] Alsaab HO, Sau S, Alzhrani R, et al. PD-1 and PD-L1 Checkpoint Signaling Inhibition for Cancer Immunotherapy: Mechanism, Combinations, and Clinical Outcome. Front Pharmacol, No.8, pp. 561, 2017.

[26] Syn NL, Teng MWL, Mok TSK, et al. De-novo and acquired resistance to immune checkpoint targeting. Lancet Oncol, Vol. 18, No.12, pp. e731-e741, 2017.

[27] Sunshine J, Taube JM. PD-1/PD-L1 inhibitors. Curr Opin Pharmacol, No. 23, pp. 32-38, 2015.

[28] Achrol AS, Rennert RC, Anders C, et al. Brain metastases. Nat Rev Dis Primers, Vol. 5, No, 1, pp. 5, 2019.

[29] Langer CJ, Mehta MP. Current management of brain metastases, with a focus on systemic options. J Clin Oncol, Vol. 23, No. 25, pp. 6207-6219, 2005.

[30] Pardoll DM. The blockade of immune checkpoints in cancer immunotherapy. Nat Rev Cancer, Vol. 12, No. 4, pp. 252-264, 2012.

[31] Fehrenbacher L, Spira A, Ballinger M, et al. Atezolizumab versus docetaxel for patients with previously treated non-small-cell lung cancer (POPLAR): a multicentre, open-label, phase 2 randomised controlled trial. Lancet, Vol. 387, No. 10030, pp. 1837-1846, 2016.

[32] Horn L, Spigel DR, Vokes EE, et al. Nivolumab Versus Docetaxel in Previously Treated Patients With Advanced Non-Small-Cell Lung Cancer: Two-Year Outcomes From Two Randomized, Open-Label, Phase III Trials (CheckMate 017 and CheckMate 057). J Clin Oncol, Vol. 35, No. 35, pp. 3924-3933, 2017. 